\title{
Reviewing the evidence: do orthodontic premolar extractions directly cause obstructive sleep apnea?
}

\begin{abstract}
Objectives: The aim of this paper is to review recent literature on the effects of orthodontic premolar extractions contributing to obstructive sleep apnea. The paper also reviews the literature regarding effects of extractions on dental arch length, total tongue space changes, and upper airway changes.
\end{abstract}

Methods: The PubMed database was systematically searched to obtain all the current eligible papers, covering the period from 2008-2017. Exclusion criteria included papers not published in the English language. Searches included reference key words including: obstructive sleep apnea, orthodontic treatment, premolar extraction, and airway space.

Results: After assessing the available articles, a total of 15 articles were chosen in the correct time frame to be considered a current analysis of the data.

Conclusion: To date there is no evidence that orthodontic premolar extractions directly cause obstructive sleep apnea.

Keywords: obstructive sleep apnea, orthodontic treatment, premolar extraction, airway space
Volume 8 Issue I - 2017

JD English, A Rodgers

UT Health School of Dentistry Houston TX, USA

Correspondence: JD English, The University of Texas School of Dentistry, Chair and Graduate Program Director, Department of Orthodontics, 7500 Cambridge Suite \#5130, Houston, TX 77054, USA, Tel (7I3) 486 - 4I27,

Email Jeryl.D.English@uth.tmc.edu

Received: May 23, 2017| Published: July 10, 2017
Abbreviations: OSA, obstructive sleep apnea; TAL, total airway length; CBCT, cone beam computed tomography; OP, oropharynx/oropharyngeal; NP, nasopharynx/nasopharyngeal; 2D, two dimensional; 3D, three dimensional

\section{Introduction}

Obstructive sleep apnea (OSA) can be a serious and potentially fatal breathing disorder if left untreated leading to heart arrhythmias, heart failure, high blood pressure, stroke and diabetes. ${ }^{1-5}$ This disorder causes a person to experience pauses in breathing due to collapse or blockage of the upper airway during sleep. ${ }^{3,5}$ The true cause of OSA can be one or a multiple of the following: the size and position of soft and hard tissue structures of the oro-facial complex, tongue size, soft palate thickness and length, adenotonsillar size, hyoid bone position, short mandibular length, high mandibular plane angle (dolichofacial growth pattern), Class II skeletal and dental malocclusions, and a narrow maxillary arch width, among others. ${ }^{5}$

Previously within the literature, it has been questioned whether orthodontic treatment with the extraction of 4 premolars causes a reduction of dental arch length with a subsequent reduction or crowding of the resting tongue space, promotion of upper airway collapse during sleep and subsequently, could contribute to OSA. ${ }^{5-9}$ Due to the fact that there is an abundance of sleep medicine practitioners or "non-extraction" orthodontists who believe that the use of extraction therapy in orthodontic treatment causes obstructive sleep apnea, it is important to discuss the evidence available to ensure that patients receive appropriate and suitable treatment based on their needs. ${ }^{5}$

After reviewing 15 articles selected from the recent literature, we assessed whether the occurrence of dental arch reduction after orthodontic premolar extractions has been found to be a potential cause of obstructive sleep apnea. We also reviewed whether extractions alter the size of the upper airway due to changes in the pharyngeal airway and the total tongue space after orthodontic retraction of the upper and lower anterior teeth.

\section{Discussion}

Throughout the years, studies have questioned whether or not orthodontic premolar extractions contribute to respiratory blockage and OSA, but to date, research efforts have not been able to confirm a positive correlation. On the contrary, efforts lean towards no relationship between the extraction of four premolars and the incidence of OSA. It should be noted though, that in a select few studies, the change in airway space following orthodontic extraction of four premolars differed according to the severity of the dental crowding, the amount of protrusion of the anterior dentition and the variety of the mechanics utilized to close the extraction spaces. ${ }^{6,-14}$

In patients with significantly crowded arches, or those with a bimaxillary protrusive dentition, one avenue of orthodontic treatment may include premolar extractions (frequently one premolar per quadrant is extracted). Bimaxillary proclination of the maxillary and mandibular incisors would necessitate a reduction in the proclination of the anterior teeth, consequently decreasing soft-tissue procumbency and convexity. Treatment for this malocclusion can include the extraction of four first premolars and retraction of the maxillary and mandibular anterior teeth. ${ }^{6}, 15$ In significantly crowded arches, extractions are performed to alleviate the crowding and further align the dentition.

It is then important to look at studies that review the relationship between OSA and dental extractions for orthodontic means. A study of four first premolar extractions in bimaxillary protrusive individuals did not show a reduction of the upper airway space (pharyngeal volume) despite a reduction in intermolar width (an expected occurrence after the process of premolar extraction and reciprocal space closure) and a decrease in tongue space. ${ }^{6}$

A recent study, in which medical and dental electronic health records were compiled and analyzed, was carried out to determine whether four premolar extractions caused OSA. This is one of a few studies that utilizes polysomnography to determine the presence or absence of an OSA diagnosis. Evidence from these records could not support a causative relationship. ${ }^{5}$ 
An earlier study underwent a retrospective analysis to compare pre- and post-treatment cephalometric radiographs to observe the mean differences in nasopharyngeal dimensions and total airway length (TAL). No statistical significance was evident between the pre- and post-treatment groups. ${ }^{7}$ A speculated reason for this could be due to the fact that the tongue position during total retraction of anterior teeth during orthodontic treatment does not affect the TAL or nasopharyngeal dimensions. But it was observed that after extraction treatment, the velopharyngeal, glossopharyngeal, and hypopharyngeal airways became narrower following orthodontic therapy. ${ }^{7}$ Another study also reported a decrease in velopharyngeal and glossopharyngeal airway spaces after full retraction of the incisors. ${ }^{12}$ It is important to note that a $2 \mathrm{D}$ evaluation via cephalometric analyses, although previously accepted as an acceptable method, is still very limited and would be further supported with 3D evaluations to assess total airway space. In contrast to the previous study, Valiathan et al., ${ }^{9}$ concluded that two separate groups, an extraction and a non-extraction group, showed no significant difference in oropharyngeal (OP) airway space from initial treatment to final treatment. This study utilized both lateral cephalometry and cone beam $\mathrm{CT}(\mathrm{CBCT}){ }^{9}$

\section{Conclusion}

To date there is no evidence that orthodontic premolar extractions directly cause obstructive sleep apnea. A considerable number of studies have analyzed the upper airway dimensions either via cephalometry or CBCT, but there is very limited information available on the relationship between the presence of orthodontic premolar extractions, respiratory function and obstructive sleep apnea8. Therefore, it is crucial to understand that even if premolar extractions and incisor retraction did decrease certain dimensions within the upper airway, this does not provide evidence that such a decrease would predispose the airway to collapse, hence leading to incidences of OSA. There is a definite need for further 3D studies that utilize diagnostic and assessment methodologies while measuring direct locations of airway obstructions during sleep.

Additional studies are required to review the actual functional assessment of breathing after premolar extractions and a further correlation between adolescent individuals and adults. When determining contributors towards OSA, it is important to account for growth and determine skeletal discrepancies prior to orthodontic treatment.

\section{Acknowledgements}

Fred \& Dianne Garrett Endowment.

\section{Conflicts of interest}

No financial interest or any conflict of interest exists.

\section{Funding}

None.

\section{References}

1. Tung P, Levitzky YS, Wang R, et al. Obstructive and Central Sleep Apnea and the Risk of Incident Atrial Fibrillation in a Community Cohort of Men and Women. Journal of the American Heart Association. 2017;6(7):e004500.

2. Drager LF, Polotsky VY, Lorenzi-Filho G. Obstructive Sleep Apnea: An Emerging Risk Factor for Atherosclerosis. Chest. 2011;140(2):534-542.

3. Punjabi NM, Caffo BS, Goodwin JL, et al. Sleep-Disordered Breathing and Mortality: A Prospective Cohort Study. Plos Med. 2009;6(8):e1000132.

4. Shaw JE, Punjabi NM, Wilding JP, et al. Sleep Disordered Breathing and Type 2 Diabetes - A Report from the International Diabetes Federation Taskforce on Epidemiology and Prevention. Diabetes Res Clin Pract. 2008;81(1):2-12.

5. Larsen AJ, Rindal DB, Hatch JP, et al. Evidence Supports No Relationship between Obstructive Sleep Apnea and Premolar Extraction: An Electronic Health Records Review. J Clin Sleep Med. 2015;11(12):1443-1448.

6. Emad Al M, Nizar El S, Abu A. First premolar extraction effects on upper airway dimension in bimaxillary proclination patients. Angle Orthod. 2012;82(5):853-859.

7. Sharma K, Shrivastav S, Sharma N, et al. Effects of first premolar extraction on airway dimensions in young adolescents: A retrospective cephalometric appraisal. Contemp Clin Dent. 2014;5(2):190-194.

8. Hu Z, Yin X, Liao J, et al. The effect of teeth extraction for orthodontic treatment on the upper airway: a systematic review. Sleep Breath. 2015;19(2):441-451.

9. Valiathan M, El H, Hans MG, et al. Effects of extraction versus nonextraction treatment on oropharyngeal airway volume. Angle Orthod. 2010;80(6):1068-1074.

10. Stefanovic N, El H, Chenin DL, et al. Three-dimensional pharyngeal airway changes in orthodontic patients treated with and without extractions. Orthod Craniofac Res. 2013;16(2):87-96.

11. Wang Q, Jia P, Anderson NK, et al. Changes of pharyngeal airway size and hyoid bone position following orthodontic treatment of Class I bimaxillary protrusion. Angle Orthod. 2012;82(1):115-121.

12. S Bhatia, B Jayan, SS Chopra. Effect of retraction of anterior teeth on pharyngeal airway and hyoid bone position in Class I bimaxillary dentoalveolar protrusion. Med $J$ Armed Forces India. 2016;72(Suppl 1):S17-S23.

13. Germec-Cakan D, Taner T, Akan S. Uvulo-glossopharyngeal dimensions in non-extraction, extraction with minimum anchorage, and extraction with maximum anchorage. Eur J Orthod. 2011;33(5):515-520.

14. V Katyal, D Kennedy, J Martin, et al. Paediatric sleep-disordered breathing due to upper airway obstruction in the orthodontic setting: a review. Aust Orthod J. 2013;29(2):184-192.

15. Chen Y, Hong L, Wang CL, et al. Effect of large incisor retraction on upper airway morphology in adult bimaxillary protrusion patients: Three-dimensional multislice computed tomography registration evaluation. Angle Orthod. 2012;82(6):964-970. 